\title{
Fat grafting versus implant-based treatment of breast asymmetry, a single surgeon experience over 13 years: a paradigm shift?
}

\author{
Pietro G. di Summa ${ }^{1,2}$, Rik Osinga ${ }^{1,3}$, Gianluca Sapino ${ }^{2}$, Katie Glen ${ }^{1}$, Gillian Higgins ${ }^{1}$, \\ Sherylin Tay ${ }^{1}$, Eva Weiler-Mithoff ${ }^{1}$
}

${ }^{1}$ Canniesburn Plastic Surgery Unit, Glasgow Royal Infirmary, Glasgow, UK; ${ }^{2}$ Department of Plastic, Reconstructive and Aesthetic Surgery, University Hospital of Lausanne (CHUV), Lausanne, Switzerland; ${ }^{3}$ Department of Plastic, Reconstructive and Aesthetic Surgery, University Hospital of Basel, Basel, Switzerland

Contributions: (I) Conception and design: PG di Summa, R Osinga, G Sapino; (II) Administrative support: All authors; (III) Provision of study materials or patients: E Weiler-Mithoff, PG di Summa; (IV) Collection and assembly of data: G Sapino, PG di Summa; (V) Data analysis and interpretation: G Sapino, PG di Summa; (VI) Manuscript writing: All authors; (VII) Final approval of manuscript: All authors.

Correspondence to: Pietro G. di Summa, MD, MD, PhD, FEBOPRAS, FMH (Plast.). Consultant Plastic and Reconstructive Surgeon, Department of Plastic and Hand Surgery, Centre Hospitalier Universitaire Vaudois (CHUV), Rue du Bugnon 46, 1011 Lausanne, Switzerland. Email: pietro.disumma@chuv.ch.

\begin{abstract}
Background Breast asymmetry can result from congenital or traumatic aetiologies. Breast implants, autologous fat grafting, or a combination of both of these techniques are commonly used to achieve symmetry. This study adds critical evaluation of long-term patient outcomes in a large study group, to evaluate pearls and pitfalls of these treatment modalities.

Methods: A prospectively maintained database of a single surgeon experience in breast asymmetry treatment over a 13-year period (2006-2018) was retrospectively analysed. Breast implant surgery and fat grafting to treat asymmetry were compared in terms of number of operations to achieve symmetry, complications, and overall patient satisfaction.

Results: Thirty-five patients underwent breast implant surgery, requiring an average $2.1 \pm 1.6$ operations to achieve symmetry, with a major complication rate (requiring secondary procedures) of $26 \%$ ( $\mathrm{n}=9$ ). Again, $26 \%(n=9)$ were converted to lipofilling due to either implant removal or unsatisfactory results. Thirty $(86 \%)$ patients underwent fat transfer monotherapy to achieve symmetry and no major complications were recorded. Nine percent $(n=3)$ of these patients preceded to have additional implant surgery.

Conclusions: Although implant-based reconstruction seemingly offers a quick single stage procedure, it is associated with significantly more revision procedures as a result of complications including capsular contracture, implant rupture and breast distortion. Fat grafting, despite requiring sequential operations to achieve initial symmetry, ultimately offers a more durable result and is associated with significantly fewer and more minor complications, while not increasing the total number of procedures required to achieve symmetry in the long term.
\end{abstract}

Keywords: Breast asymmetry; congenital asymmetry; tuberous breast; fat grafting; breast implant

Submitted Feb 11, 2021. Accepted for publication Apr 26, 2021.

doi: $10.21037 / g s-21-91$

View this article at: https://dx.doi.org/10.21037/gs-21-91

\section{Introduction}

Breast asymmetry can include both shape and volume of the gland can be classified as congenital or acquired and has a significant physical and psychological impact on women (1).
According to literature, some degree of congenital breast asymmetry is very common in patients who are seeking breast augmentation procedures and can involve as much as $90 \%$ of cases (2). 
Tuberous breast deformity (TBD) represents a specific type of congenital breast asymmetry where an alteration of the superficialis fascia, especially at the lower quadrants of the breast, limits the physiological expansion of breast mound (3) and thus its shape during growth. Features of tuberous breasts include a small breast, cylindrical shape with a deficient diameter of the mammary base, hypoplasia especially of the lower quadrants, abnormal elevation of the inframammary fold, breast tissue herniation into the areolar region; and sometimes increased areolar diameter (3-5). It presents in various degrees of severity and can be unilateral or bilateral.

The etiology of TBD remains vague. Similarly, TBD classifications are multiple and can be rather confusing. According to Grolleau et al., type I is hypoplasia of the medial lower quadrant, type II is hypoplasia of both lower quadrants and type III is hypoplasia of the four quadrants with severe breast constriction $(3,6)$. In the literature type $\mathrm{I}$ is the most frequent deformation (3). However, as noted by Delay et al. (7), types II and III are the ones frequently treated as these present the more obvious and significant deformity.

Secondary, non-oncological causes of breast asymmetry include trauma influencing breast shape, burns and iatrogenic surgical causes such as thoracotomies in childhood (8). Breast asymmetry, and particularly TBD, is said to be one of the most challenging breast conditions to treat surgically (4). Two main surgical options exist, which can be used independently but are commonly combined to achieve optimum results; fat grafting and breast implants.

Fat transfer to improve volume, projection, shape and contour is commonly used for both aesthetics and reconstructive surgery of the breast (7). The main challenge with fat grafting in congenital asymmetric, and especially in tuberous breasts, is the presence of fibrous tissue which makes fat grafting more difficult to perform. Moreover, in very slender patients, fat grafting donor sites may be limited. It is also necessary for the patients to maintain their weight as this can affect the result over time. Fat grafting represents a generally safe technique (with the exception of rare complications such as pneumothorax and fat embolism) (9). More common complications such as palpable nodules from fat necrosis, cyst formation, calcification and donor site irregularities, due to overtreatment or technical errors, may occur during the learning curve. Infections and overexpected fat resorption affect especially smokers, with final low volume gain (9).

Implant based reconstruction can theoretically achieve correction of asymmetry in one stage, addressing volume and shape, especially when gland is remodeled over the implant (10). Despite being theoretically the preferred option when treating asymmetry in patients with lower body mass indexes (BMIs) (11), implants retain some major disadvantages. Animation, change of shape due to capsular contracture (2.8-45\% of women), and need for implant exchange over time, potentially commit the patient to lifelong interventions (12-14). Moreover, implants pose the risk of potential complications which are difficult to treat, such as implant shift or malrotation, double-bubble deformity, implant infection, nipple-areola complex (NAC) asymmetry and distortion. These complications further increase the total cost of this type of reconstruction, with subsequent consequences for the patient and the Health Care System. Finally, the risk of anaplastic large-cell lymphoma (ALCL) associated with breast reconstruction using textured implants must be considered and the patient counselled appropriately (15).

This study retrospectively analyses an extensive singlesurgeon patient database of consecutive patients, comparing patient populations with a similar degree of breast asymmetry over a 13-year time period. Long-term outcomes and complications of both implant-based and fat grafting procedures are discussed, to compare these techniques for reconstruction of breast asymmetry. This large series aims to contribute to the decision-making process of the reconstructive surgeon, highlighting treatment pathways for individual patient situations in the complex domain of breast asymmetry. We present the following article in accordance with the STROBE reporting checklist (available at https://dx.doi.org/10.21037/gs-21-91).

\section{Methods}

All participants were adult female patients with breast asymmetry who underwent surgical treatment by the senior author between 2006 and 2018 at the Canniesburn Plastic Surgery and Burns Unit. Data was prospectively maintained and retrospectively investigated. All patients with congenital breast asymmetry (e.g., Poland syndrome, idiopathic, TBD type II and III) and post-traumatic/secondary breast asymmetry were included and divided into two treatment groups: patients treated primarily with implant-based surgery and patients treated primarily by fat grafting (Table 1).

Data that was not readily present in the database, was obtained by operative notes, discharge letters, clinic letters and anesthetic charts. 
Table 1 Patient's characteristics

\begin{tabular}{|c|c|c|}
\hline Characteristics & Implant based & Fat grafting \\
\hline Number of patients & 35 & 30 \\
\hline \multicolumn{3}{|l|}{ Etiology } \\
\hline Congenital deformity & 34 & 28 \\
\hline Poland & 2 & 1 \\
\hline Idiopathic & 26 & 7 \\
\hline Tuberous breast(s) & 6 & 20 \\
\hline Traumatic & 1 & 2 \\
\hline \multicolumn{3}{|l|}{ Side } \\
\hline Unilateral & 7 & 14 \\
\hline Bilateral & 28 & 16 \\
\hline \multicolumn{3}{|l|}{ Age (years) } \\
\hline Mean & $28 \pm 10$ & $33 \pm 11$ \\
\hline Range & $19-57$ & $18-52$ \\
\hline \multicolumn{3}{|l|}{ BMI $\left(\mathrm{kg} / \mathrm{m}^{2}\right)$} \\
\hline Mean & $22 \pm 2$ & $24 \pm 3$ \\
\hline Range & $18-24$ & 20-33 \\
\hline \multicolumn{3}{|l|}{ Comorbidities } \\
\hline Smoking & 3 & 3 \\
\hline Diabetes & 0 & 0 \\
\hline
\end{tabular}

BMI, body mass index.

Exclusion criteria included patients below the age of 16 years, BMI $>27 \mathrm{~kg} / \mathrm{m}^{2}$, asymmetry inferior to 2 cup sizes, moderate TBD (grade I), incomplete or non-available data, asymmetry due to oncological surgery or treated with therapeutic reduction mammoplasty only.

The type and number of procedures, patient's age at the time of first procedure, BMI, smoking habit, presence of diabetes and other relevant comorbidities at the time of first surgical treatment were recorded. Both conservatively and surgically treated postoperative complications were sought as the number and type of additional or complementary surgeries (e.g., mastopexies or revision surgeries) required until end of treatment. We considered major complications those requiring an additional surgical operation (e.g., postoperative bleeding, implant rupture, painful capsulitis, etc.). In outpatient clinic letters of the senior author, symmetry achievement outcomes were evaluated based on surgeon examination findings, patient verbal feedback and patient desire for further surgeries. Patients whose symmetry outcomes were uncertain or not specified, were included in the study concerning patient population, complications, number of procedures and operative details, but were excluded from the analysis of patient satisfaction outcomes.

The study was conducted in accordance with the Declaration of Helsinki (as revised in 2013). Informed consent was obtained from all patients, including approval for photographic/video documentation. No need for local ethical committee approval because of the retrospective nature of the study.

\section{Surgical technique}

\section{Implant based procedure}

All procedures were performed under general anesthesia with a prophylactic dose of intravenous (IV) antibiotics at induction. All patients were preoperatively examined in an upright position to identify the existing and estimated future inframammary fold. Furthermore, the position of the corrected NAC was marked. All but one augmentation used an inframammary fold incision for implant placement. All implants were placed in the retro glandular plane. When simple augmentation was not sufficient to re-establish symmetry (e.g., tuberous breast and deficit of the lower pole) the Ribeiro technique of glandular reshape was used (10). After all procedures a wound tape was placed on the suture line for 3 months.

\section{Autologous fat grafting}

All procedures were performed under general anesthesia with a prophylactic dose of IV antibiotics at induction. We followed the protocol previously described by the senior author (EWM) (16) but without the use of the Celutions system (Cytori Therapeutics; San Diego, CA, USA) as we did not enrich our grafts with adipose-derived stem cells. Prior to harvest, the volume of the defect and required graft were visually estimated by the surgeon. Roughly twice the intended volume of graft was harvested using standard tumescent, syringe-based liposuction. The graft was centrifuged at 3,000 rpm for 3 minutes. Discarding the washing fluid left approximately $65 \%$ of the volume to be transferred through $2 \mathrm{~mm}$ stab incisions outside the region of the breast defect. Graft tissue was deposited in the subcutaneous plane, trying to avoid the breast tissue itself. The fat delivery was performed in a multi-planar, multidirectional fan-shaped technique to maximize the surface area of the graft to the native, well perfused tissue. After 
Table 2 Outcomes and complications

\begin{tabular}{|c|c|c|}
\hline Variable & Implant group $(n=35)$ & Fat grafting group $(n=30)$ \\
\hline Converted to implant & - & $3(10 \%)$ \\
\hline Converted to fat grafting & $9(26 \%)$ & - \\
\hline Fat injected & - & $344 \pm 161$ cc (hypoplastic side) \\
\hline \multirow[t]{2}{*}{ Implant sizes } & $277 \pm 70$ cc (hypoplastic side) & - \\
\hline & $193 \pm 110$ cc (contralateral side) & \\
\hline \multirow[t]{2}{*}{ Patient satisfaction } & $24 / 35(69 \%)$ & $24 / 30(80 \%)$ \\
\hline & $31 / 35(86 \%)$ after conversion & $27 / 30(90 \%)$ after conversion \\
\hline \multirow[t]{5}{*}{ Minor complications } & 4 pain/dysesthesia & 4 donor site irregularity \\
\hline & 3 reduced nipple-areolar complex sensation & 2 pain at donor site \\
\hline & 1 hypertrophic scar & 1 self-resorbing lymphocele \\
\hline & 1 wound dehiscence & 1 oil cyst at the recipient site \\
\hline & 3 palpable implant/wrinkling & \\
\hline
\end{tabular}

graft delivery, the patient was placed in a sitting position to examine the result and additional injections were performed if necessary.

\section{Statistical analysis}

Patient groups were compared using independent two-sided $t$-tests for means, Mann-Whitney $U$ tests for medians and two-sided Chi-square or Fisher's exact test as appropriate in order to analyze categorical variables. We verified the assumption of normality using the Shapiro-Wilk test. Statistical significance was set at a $\mathrm{P}$ value $<0.05$. Statistical analysis was performed using GraphPad Prism (version 8.0, GraphPad software, La Jolla, CA, USA).

\section{Results}

Please refer to Table 2 for outcomes and complications data of both groups.

\section{Implant-based group}

37 consecutive patients benefited of implants to solve breast asymmetry. Of these, 2 were excluded due to incomplete documentation (final $\mathrm{n}=35$ ). The follow-up was on average $33 \pm 27$ months (12-101 months). The mean age was $28 \pm 10$ years (mean $\pm \mathrm{SD}$ ) at time of first surgical treatment, the BMI was $22 \pm 2 \mathrm{~kg} / \mathrm{m}^{2}$ (mean $\pm \mathrm{SD}$ ). Thirtyfour out of 35 patients ( $97 \%$ ) suffered from congenital asymmetry (2 Poland syndrome, 26 idiopathic, 6 from tuberous breasts), 1 patient only had secondary asymmetry from trauma (Figure 1).

A bilateral breast augmentation was performed in 28 patients $(80 \%)$, while in 7 patient $(20 \%)$ a monolateral implant on the hypoplastic side was sufficient to correct the asymmetry between the two breast mounds. When monolateral breast asymmetric treatment was performed, permanent expandable breast implants (Becker) were used, while in the bilateral augmentations silicone gel implants were used. A breast lift was associated with the augmentation procedure in 4 patients (11\%) (in 2 cases bilaterally). The mean implant volume used for the hypoplastic side was $277 \pm 70 \mathrm{cc}$ (mean \pm SD) (range, 160-400) while on the contralateral side was $193 \pm 110 \mathrm{cc}$ (mean \pm SD) (range, 100-400). The differences in implant size among sides were, as expected, statistically significant 

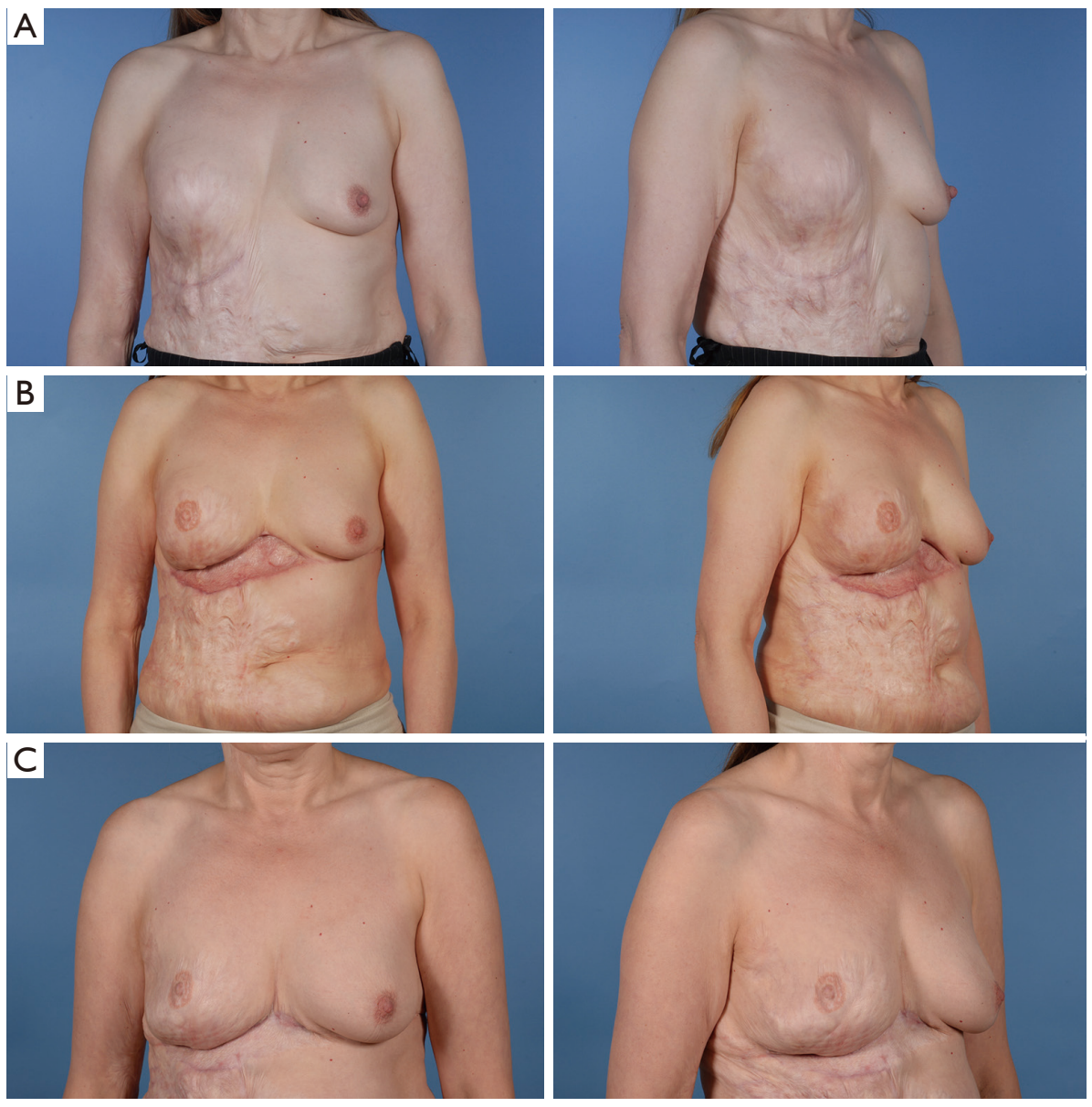

Figure 1 Case No. 1. A 50-year-old patient with burn trauma at the right breast during infancy underwent right breast reconstruction with implant (A). The right inframammary fold was reconstructed using a left thoraco-epigastric adipo-cutaneous flap (B). A few years later, the implant was removed due to intracapsular rupture and the breast was successfully reconstructed using autologous fat transfer (2 operations required, total fat injected: $350 \mathrm{cc})(\mathrm{C})$.

$(\mathrm{P}<0.001)$. On average, each patient underwent $2.1 \pm 1.6$ (mean $\pm \mathrm{SD}$ ) procedures (range, $1-6$ ), which was significantly higher compared to the fat graft group $(\mathrm{P}<0.05)$.

Among complications, in 9 cases (26\%), supplementary procedures were necessary due to prosthesis-related complications such as ruptures and capsular contracture, requiring final implant removal in 6 patients $(17 \%)$ and implant exchange in the other 3 (9\%).

Five out of 6 patients who had implants permanently removed, where treated by fat grafting to regain shape and volume. Further 4 patients who were not satisfied with shape and/or volume after implant only treatment benefited of supplementary fat graft. Taken together, 9 patients $(26 \%)$ of the implant-based group were addressed to fat grafting to correct either volume, shape lack or as a total alternative to removed implants. In those patients, $297 \pm 293 \mathrm{cc}$ of fat tissue where injected on average, in $1.7 \pm 0.6$ additional surgical procedure (all expressed as mean $\pm \mathrm{SD}$ ).

When considering initial implant treatment only 24 out of 35 patients $(69 \%)$ achieved stable symmetry and were satisfied with the result at the last follow-up (average $33 \pm 27$ months). Among the patients who secondarily underwent fat grafting because not initially satisfied (24\% of the group), 7 out of 9 achieved a satisfactory result (raising the total satisfaction rate of $86 \%$ ). Among these, 4 out of the 5 patients who underwent final implant removal and total conversion to fat grafting, considered themselves satisfied with the final results.

Relatively minor complications, not requiring implant removal or supplementary procedures including pain/ 

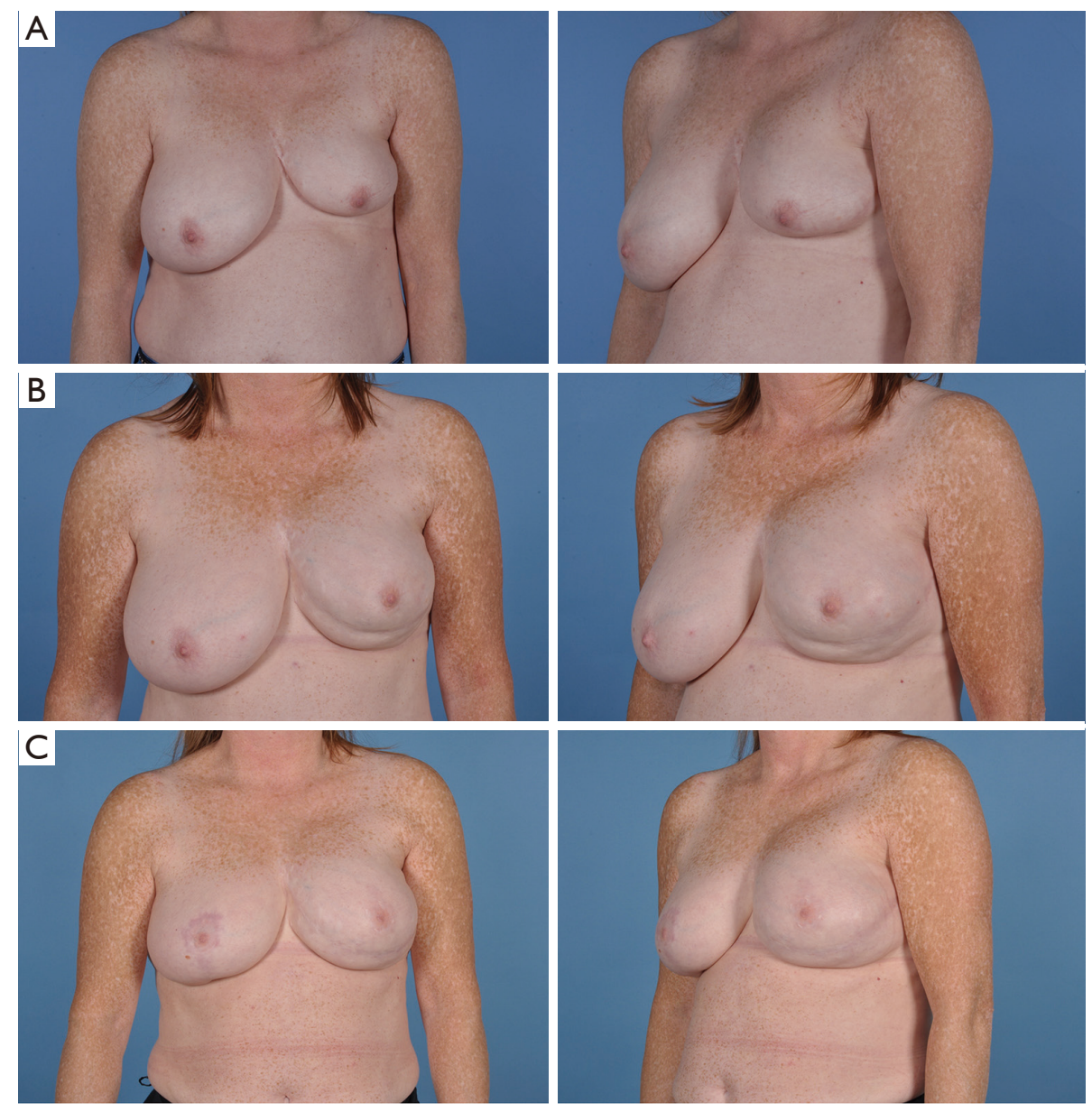

Figure 2 Case No. 2. A 46-year-old patient suffering from breast asymmetry L < R (A). Satisfactory result was achieved with 3 tissue fat transfers to the left breast (total fat injected: $700 \mathrm{cc}$ ) (B) and a right breast reduction performed during the last lipo-transfer session $(\mathrm{C})$.

dysesthesia $(n=4)$, reduced NAC sensation $(n=3)$, hypertrophic scar $(n=1)$, wound dehiscence $(n=1)$ and palpable implant/wrinkling $(\mathrm{n}=3)$.

\section{Fat grafting group}

Thirty-four patients were primarily treated with autologous fat transfer. Of those, 4 were excluded due to incomplete documentation, resulting in 30 patients included in the study with a mean follow-up of $25 \pm 17$ months (12-71 months). Age at time of first surgical treatment was $33 \pm 11$ years (mean $\pm \mathrm{SD}$ ) the BMI was $24 \pm 3 \mathrm{~kg} / \mathrm{m}^{2}$ (mean $\pm \mathrm{SD}$ ). Both of these values were statistically significantly different when compared to the implant treatment group $(\mathrm{P}<0.05$ and $\mathrm{P}<0.001$, respectively). Twenty-eight patients suffered from congenital deformity (12 unilateral, 16 bilateral): 1 Poland Syndrome,
7 idiopathic, 20 tuberous breasts and 2 from burn-related trauma. Twenty patients received one fat grafting session only, 7 patients had it twice and 3 patients benefited of the procedure three times (Figure 2).

On average, to reach a final satisfactory result, every patient underwent $1.5 \pm 0.8$ (mean \pm SD) fat grafting procedures, which was statistically significantly lower when compared to the implant group $(\mathrm{P}<0.05)$. The average amount of fat tissue totally harvested for each patient, including multiple procedures, was $780 \pm 490 \mathrm{cc}$ (mean \pm SD), (range, 200-2,560 cc). After processing of the adipose tissue, the hypoplastic side benefitted in average of the injection of $344 \pm 161 \mathrm{cc}($ mean $\pm \mathrm{SD}$ ) (range, 140-750 cc) while the contralateral breast was injected in 16 patients $(53 \%)$ with in average $163 \pm 159 \mathrm{cc}($ mean \pm SD) (range, $60-500 \mathrm{cc}$ ). As found in the implant group, the differences 
Table 3 TBD patients characteristics and outcomes

\begin{tabular}{|c|c|c|}
\hline Variable & TBD implant & TBD fat \\
\hline Mean age, years & $29 \pm 9$ & $35 \pm 10$ \\
\hline Mean BMI $\left(\mathrm{kg} / \mathrm{m}^{2}\right)$ & $23 \pm 1$ & $25 \pm 2$ \\
\hline Mean follow-up & $30 \pm 22$ & $23 \pm 10$ \\
\hline Converted to fat grafting & $2(33 \%)$ & - \\
\hline Converted to implant & - & $1(5 \%)$ \\
\hline \multirow[t]{2}{*}{ Fat injected } & \multirow[t]{2}{*}{-} & $339 \pm 140$ cc (hypoplastic side) \\
\hline & & $241 \pm 140$ cc (contralateral side) \\
\hline Patients satisfaction before conversion & $66 \%$ & $90 \%$ \\
\hline Additional procedures & 2 Benelli pexy, 1 breast reduction & 2 Benelli pexy \\
\hline \multirow[t]{4}{*}{ Complications } & 1 implant rupture and explantation & 1 oil cyst \\
\hline & 1 capsulitis & 2 volume irregularities \\
\hline & 1 nipple loss of sensation & 1 donor site lymphocele \\
\hline & 1 wound dehiscence & \\
\hline
\end{tabular}

TBD, tuberous breast deformity.

of injected fat among sides were, as expected, statistically significant $(\mathrm{P}<0.001)$. Due to shape and volume asymmetry on the contralateral side, 3 monolateral mastopexy (10\%) and 1 reduction mammaplasty (3\%) were performed simultaneously to the first lipofilling. In 3 patients a combined procedure with prosthesis was necessary to achieve the requested breast volume and shape (all bilateral). In one of these patients the treatment was planned as "hybrid" from the beginning, while two patients opted for implants placement after initial fat grafting in order to reach bigger breast size and improve symmetry. Twentyfour patients $(80 \%)$ reached a satisfactory symmetry result thanks to lipofilling alone while in the aforementioned 3 patients a combined breast augmentation procedure was necessary (for a final satisfactory symmetry achievement on $90 \%$ of the cases). As mentioned, in the previous paragraph, 9 patients originally treated with implant were converted to fat graft in reason of multiple prosthesis complications or unsatisfactory results.

When including all 39 patients who received some sort of fat grafting (30 as primary procedure, 9 as "converted to fat graft"), no major complications occurred (e.g., embolism, death), neither supplementary surgeries were necessary. Seven patients (18\%) referred lipofilling related complications including irregularity $(n=4)$, pain $(n=2)$ and self-resorbing lymphocele $(\mathrm{n}=1)$ at donor site, and oil cyst $(\mathrm{n}=1)$ at the recipient site.

\section{Tuberous breasts}

A sub-group analysis focusing on tuberous breasts only was performed to identify differences in outcomes between the two techniques (Table 3).

In the implant group we could include 6 TBD patients with a mean age of $29 \pm 9$ years old, all bilateral. Two patients were converted to fat grafting (in one the implant was definitely explanted). Three patients benefited of mastopexy procedures ( 2 Benelli mastopexy and 1 reduction), average number of surgical procedures was $3 \pm 2($ mean $\pm S D)$ which resulted significantly higher compared to the fat graft group $(\mathrm{P}<0.001)$. After implant reconstructions, only $66 \%$ were satisfied with the results (4 out of 6 ), while satisfaction rose 

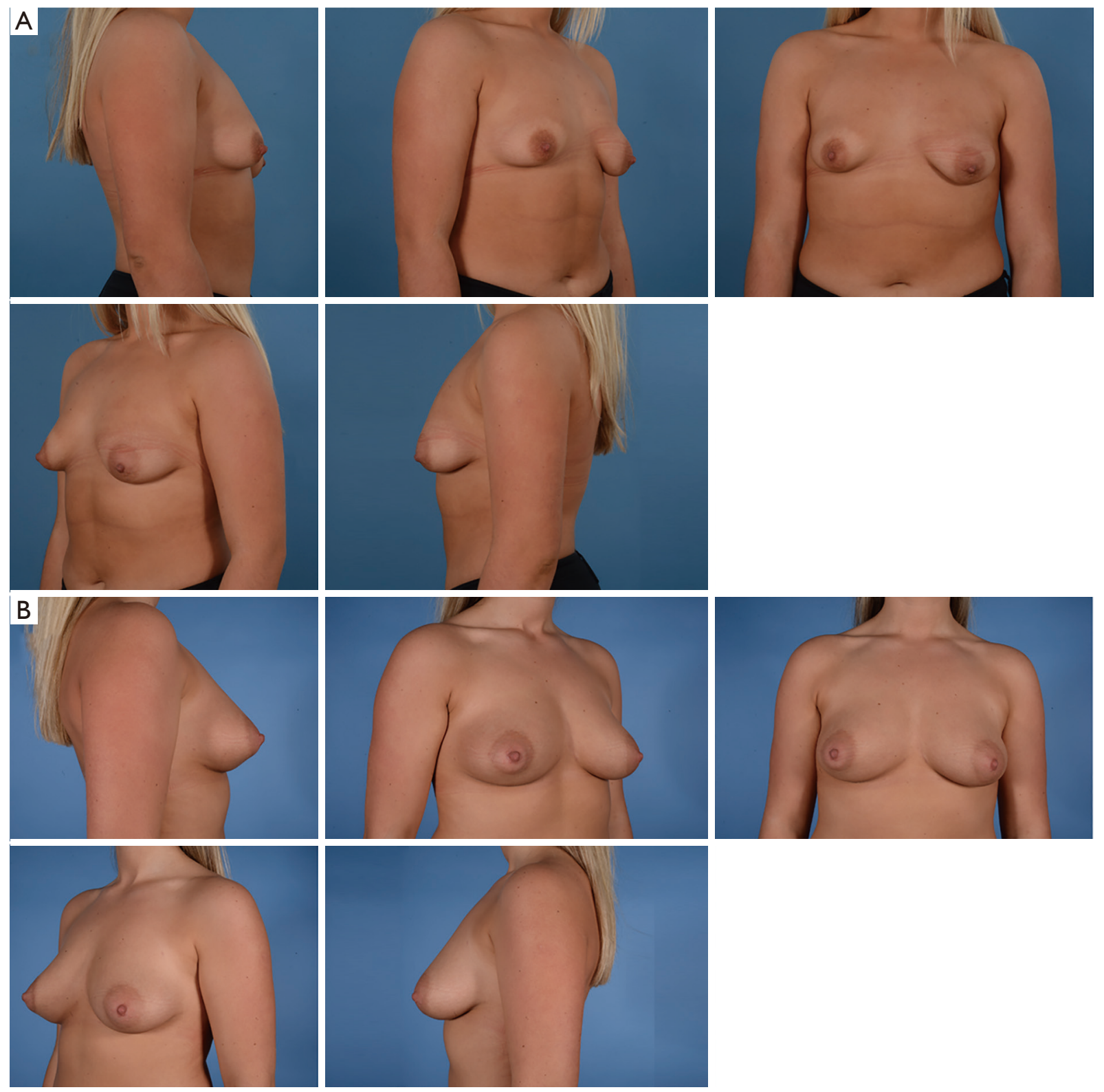

Figure 3 Case No. 3. A 25-year-old patient suffering from type II-III bilateral tuberous breast deformity (A). Satisfactory result was achieved performing 2 tissue fat transfers (total fat injection: right $350 \mathrm{cc}$, left $250 \mathrm{cc}$ ) (B).

to $100 \%$ after eventual conversion to fat graft. Among complications we could record 1 explantation due to implant rupture, 1 capsulitis with deformity (grade III according to Becker classification), 1 nipple sensation loss and 1 wound dehiscence on mastopexy.

The fat graft group included 20 patients (mean age $35 \pm 10$ years old) with TBD: 16 with bilateral and 4 with monolateral deformity (Figure 3). Fat harvested was in average $865 \pm 512 \mathrm{cc}$, fat injected $339 \pm 140$ cc (hypoplastic side) and $241 \pm 140 \mathrm{cc}$ (contralateral side), Every patient benefited in average of $1.3 \pm 0.5$ surgical procedure, with $90 \%$ satisfactory symmetry achieved (18 out of 20 ). One patient only required an implant after initial fat treatment. Two Benelli mastopexy (1 bilateral and 1 monolateral) were recorded in the group on top of fat grafting.

In terms of complications, there was 1 patient with oil 
cyst, 2 patients with volume irregularities at the donor site and 1 patient with postoperative lymphocoele at the thigh donor site.

\section{Discussion}

There is a wealth of evidence in the literature discussing the use of prosthetic and autologous treatments for breast asymmetry (17). Some studies favour implants over fat grafting, while others underline advantages of fat grafting in terms of aesthetic result, natural appearance, and stability of result over time.

This study contributes the analysis of long-term outcomes for a large patient cohort (68 patients, 109 treated breasts) with similar asymmetry features, which fit in NHS criteria of insurance covered breast asymmetry over a 12 years follow-up.

In the early years of the studied period, breast asymmetries were mainly treated with implants, using the lipofilling as a salvage tool. This attitude was progressively changed to a lipofilling based approach according to the personal experience of the senior author (EWM).

When using one technique only, data showed a significant better long-term outcome after fat grafting vs implant surgery ( $80 \%$ vs. $69 \%$ of satisfaction). Results were further improved when one technique was converted to fat grafting or was sensibly improved by fat grafting, allowing attainment of satisfactory symmetry in $90 \%$ and $86 \%$ in fat grafting and implant group, respectively.

Fat transfer seems to be particularly useful in unilateral cases for two reasons. Firstly, it is possible to inject a larger amount of fat per breast even in thinner patients; moreover, by correcting the asymmetry in an autologous way, the reconstructed breast is more prone to "age" with a natural shape similarly to the contralateral side, enabling to maintain the achieved symmetry over time.

Complication rates were higher in the implant group with $26 \%$ of the patients presenting implant-related complications. This may explain also the higher number of procedures required to achieve asymmetry in this cohort. The patients treated with fat grafting had an $18 \%$ complication rate, however these could be managed conservatively.

Our data suggests that fat grafting should be considered the mainstay in treating complex breast asymmetry cases. It can guarantee more predictable results, has lesser morbidity and accounted for lower rate of complications and higher patient satisfaction. Even patients with low BMI were successfully treated with fat gratifying in this series, illustrating that satisfactory results can be obtained in "poor donors" with refined and precise surgical planning for fat placement. We suggest addressing the lower pole and the breast base first, before attending to the NAC position, which in our series was addressed as the final step, where necessary.

Interestingly, despite the common perception that fat grafting commits the patient to a long course of multiple treatments, fat grafting provided in our casuistry satisfactory symmetry in significantly less surgical steps than the implant-based procedures, probably because the meticulous multiplanar/multidirectional technique which allowed for the maximum amount of graft take per session.

Particularly in TBD however, literature is divided among which treatment could be considered the more effective, and comparative studies are still lacking. Brault et al. compared complications and satisfaction in 37 patients suffering from TBD and treated either with lipofilling (27 breasts) or implants ( 36 breasts). According to their results, patient's satisfaction was higher in the implant group, with no differences in terms of complications and number of interventions (17). However, various published study agree that fat grafting is becoming the routine treatment for this kind of deformity as it avoids the complications related with the implants placement and responds physiologically to the breast changes over time $(6,18)$.

Our experience is consistent with the one of those authors and we think that TBD can be solved without implant use by delivering fat volume in appropriate surgical planes, when a sufficient volume of fat can be injected.

Fat grafting to improve breast asymmetry is advantageous in multiple ways: (I) the purely autologous nature of the operation, (II) the relatively low cost, (III) the reproducibility (since the technique can be repeated if required), (IV) the natural appearance and consistency of the breast postoperatively, (V) the postoperative symmetry with the contralateral breast, and, finally (VI) the secondary benefits of the liposuction involved in the procedure $(7,9)$.

With various surgical options available for correction of breast asymmetry, patients should be provided with information about different surgical options, their limitations and all possible complications. This is necessary to allow the patients to make informed decisions on their choice of procedure, to clear misconceptions that the patient may have had and to allow the patients to set realistic goals in terms of what is achievable with surgery. The keys to successful treatment are to define the nature 
of the asymmetry, respect the aesthetic goals of the patient, and perform a well thought out surgical plan (19). Timing of surgery is crucial. This is especially important in younger patient in whom the breast is still growing. If surgery is needed early, the patient and parents must be made aware of the need for revisions later, especially when implant surgery is provided. Whatever method is selected to achieve symmetry it is important for patients to be aware that the post-operative result may deteriorate over time and may require revision (19).

We need to acknowledge that this study is a retrospective investigation of two different techniques and eventual hybrid approaches of non-randomized patients, without strict quantitative assessment of aesthetic outcomes. It quantitatively demonstrates the long-term surgical complications occurring with both techniques, whilst providing qualitative evaluation of aesthetic outcomes. This study represents one of the largest series of complex consecutive breast asymmetry cases, performed by a single internationally renowned surgeon with long-term patient outcomes reported. We recommend shifting the paradigm of treatment towards the predominant use of fat grafting for improved long-term outcomes for the surgical treatment of breast asymmetry.

\section{Acknowledgments}

Funding: None.

\section{Footnote}

Reporting Checklist: The authors have completed the STROBE reporting checklist. Available at https://dx.doi. org/10.21037/gs-21-91

Data Sharing Statement: Available at https://dx.doi. org/10.21037/gs-21-91

Conflicts of Interest: All authors have completed the ICMJE uniform disclosure form (available at https://dx.doi. org/10.21037/gs-21-91). The authors have no conflicts of interest to declare.

Ethical Statement: The authors are accountable for all aspects of the work in ensuring that questions related to the accuracy or integrity of any part of the work are appropriately investigated and resolved. The study was conducted in accordance with the Declaration of Helsinki (as revised in 2013). Individual consent for this retrospective analysis, including approval for photographic \video documentation, was gathered from all patients. No need for local ethical committee approval because of the retrospective nature of the study.

Open Access Statement: This is an Open Access article distributed in accordance with the Creative Commons Attribution-NonCommercial-NoDerivs 4.0 International License (CC BY-NC-ND 4.0), which permits the noncommercial replication and distribution of the article with the strict proviso that no changes or edits are made and the original work is properly cited (including links to both the formal publication through the relevant DOI and the license). See: https://creativecommons.org/licenses/by-nc-nd/4.0/.

\section{References}

1. Grolleau JL, Pienkowski C, Chavoin JP, et al. Morphological anomalies of breasts in adolescent girls and their surgical correction. Arch Pediatr 1997;4:1182-91.

2. Rohrich RJ, Hartley W, Brown S. Incidence of breast and chest wall asymmetry in breast augmentation: a retrospective analysis of 100 patients. Plast Reconstr Surg 2003;111:1513-9; discussion 1520-3.

3. Grolleau JL, Lanfrey E, Lavigne B, et al. Breast base anomalies: treatment strategy for tuberous breasts, minor deformities, and asymmetry. Plast Reconstr Surg 1999;104:2040-8.

4. Brown MH, Somogyi RB. Surgical Strategies in the Correction of the Tuberous Breast. Clin Plast Surg 2015;42:531-49.

5. Pacifico MD, Kang NV. The tuberous breast revisited. J Plast Reconstr Aesthet Surg 2007;60:455-64.

6. Claudio Silva-Vergara C, Fontdevila J, Weshahy O. Fat Grafting Technique, A Paradigm Shift in the Treatment of Tuberous Breast. World J Plast Surg 2018;7:72-7.

7. Delay E, Guerid S. The Role of Fat Grafting in Breast Reconstruction. Clin Plast Surg 2015;42:315-23, vii.

8. Glicksman CA, Ferenz SE. The Etiologies of Chest Wall and Breast Asymmetry and Improvement in Breast Augmentation. Clin Plast Surg 2015;42:519-30.

9. Largo RD, Tchang LA, Mele V, et al. Efficacy, safety and complications of autologous fat grafting to healthy breast tissue: a systematic review. J Plast Reconstr Aesthet Surg 2014;67:437-48.

10. Ribeiro L, Canzi W, Buss A Jr, et al. Tuberous breast: a new approach. Plast Reconstr Surg 1998;101:42-50; 
discussion 51-2.

11. Tenna S, Cagli B, Brunetti B, et al. Management of Tuberous Breast Deformities: Review of Long-term Outcomes and Patient Satisfaction with BREAST-Q. Aesthetic Plast Surg 2017;41:1249-58.

12. Gabriel SE, Woods JE, O'Fallon WM, et al. Complications leading to surgery after breast implantation. N Engl J Med 1997;336:677-82.

13. Salzberg CA, Ashikari AY, Berry C, et al. Acellular Dermal Matrix-Assisted Direct-to-Implant Breast Reconstruction and Capsular Contracture: A 13-Year Experience. Plast Reconstr Surg 2016;138:329-37.

14. El-Sheikh Y, Tutino R, Knight C, et al. Incidence of capsular contracture in silicone versus saline cosmetic augmentation mammoplasty: A meta-analysis. Can J Plast Surg 2008;16:211-5.

15. Van Natta BW. Determining the True Incidence of Breast

Cite this article as: di Summa PG, Osinga R, Sapino G, Glen K, Higgins G, Tay S, Weiler-Mithoff E. Fat grafting versus implant-based treatment of breast asymmetry, a single surgeon experience over 13 years: a paradigm shift? Gland Surg 2021;10(6):1920-1930. doi: 10.21037/gs-21-91
Implant-Associated Anaplastic Large Cell Lymphoma (BIA-ALCL): The Need for Accurate Data. Aesthet Surg J 2019;39:NP230-1.

16. Pérez-Cano R, Vranckx JJ, Lasso JM, et al. Prospective trial of adipose-derived regenerative cell (ADRC)enriched fat grafting for partial mastectomy defects: the RESTORE-2 trial. Eur J Surg Oncol 2012;38:382-9.

17. Brault N, Stivala A, Guillier D, et al. Correction of tuberous breast deformity: A retrospective study comparing lipofilling versus breast implant augmentation. J Plast Reconstr Aesthet Surg 2017;70:585-95.

18. Ho Quoc C, Piat JM, Michel G, et al. Fat grafting to improve severe tuberous breast. J Gynecol Obstet Biol Reprod (Paris) 2015;44:503-9.

19. Reilley AF. Breast asymmetry: classification and management. Aesthet Surg J 2006;26:596-600. 